\title{
Neurophotonics
}

\section{Modified toolbox for optogenetics in the nonhuman primate}

Ji Dai

Ilker Ozden

Daniel I. Brooks

Fabien Wagner

Travis May

Naubahar S. Agha

Benjamin Brush

David Borton

Arto V. Nurmikko

David L. Sheinberg

\section{SPIE.}




\title{
Modified toolbox for optogenetics in the nonhuman primate
}

\author{
Ji Dai, ${ }^{a}$ Ilker Ozden, ${ }^{\mathrm{b}}$ Daniel I. Brooks, ${ }^{\text {a }}$ Fabien Wagner, ${ }^{\text {a }}$ Travis May, ${ }^{\mathrm{b}}$ Naubahar S. Agha, ${ }^{\mathrm{b}}$ Benjamin Brush, \\ David Borton, ${ }^{\mathrm{b}}$ Arto V. Nurmikko, ${ }^{\mathrm{b}, \mathrm{c}}$ and David L. Sheinberga, ${ }^{\mathrm{a}, \star}$ \\ abrown University, Department of Neuroscience, 185 Meeting Street, Box GL-N, Providence, Rhode Island 02912, United States \\ ${ }^{b}$ Brown University, School of Engineering, 182 Hope Street, Box D, Providence, Rhode Island 02912, United States \\ 'Brown University, Department of Physics, 182 Hope Street, Box D, Providence, Rhode Island 02912, United States
}

\begin{abstract}
Attracted by the appealing advantages of optogenetics, many nonhuman primate labs are attempting to incorporate this technique in their experiments. Despite some reported successes by a few groups, many still find it difficult to develop a reliable way to transduce cells in the monkey brain and subsequently monitor lightinduced neuronal activity. Here, we describe a methodology that we have developed and successfully deployed on a regular basis with multiple monkeys. All devices and accessories are easy to obtain and results using these have been proven to be highly replicable. We developed the "in-chair" viral injection system and used tapered and thinner fibers for optical stimulation, which significantly improved the efficacy and reduced tissue damage. With these methods, we have successfully transduced cells in multiple monkeys in both deep and shallow cortical areas. We could reliably obtain neural modulation for months after injection, and no light-induced artifacts were observed during recordings. Further experiments using these methods have shown that optogenetic stimulation can be used to bias spatial attention in a visual choice discrimination task in a way comparable to electrical microstimulation, which demonstrates the potential use of our methods in both fundamental research and clinical applications. () The Authors. Published by SPIE under a Creative Commons Attribution 3.0 Unported License. Distribution or reproduction of this work in whole or in part requires full attribution of the original publication, including its DOI. [DOI: 10.1117/1.NPh.2.3.031202]
\end{abstract}

Keywords: optogenetics; nonhuman primate; methodology.

Paper 15005SSR received Jan. 22, 2015; accepted for publication Apr. 9, 2015; published online May 29, 2015.

\section{Introduction}

Optogenetics has been proven to be a powerful tool to manipulate neural activity in both rodents ${ }^{1-3}$ and nonhuman primates. $^{4-10}$ In the previous period during its development in the nonhuman primate model, researchers found that they could apply this technique to modulate neural activity by light. Han et al. first successfully expressed channelrhodopsin (ChR2) in the macaque frontal cortex in $2009,{ }^{4}$ followed by another inhibitory archaerhodopsin (ArchT) in 2011. ${ }^{11}$ The same year, in 2011, another team at Stanford further characterized the safety and efficacy of optogenetics in primates. ${ }^{5}$ However, regardless of neural modulation, none of these studies have reported any behavioral effect driven by optogenetic stimulation. Starting in 2012, researchers found evidence that optogenetic stimulation in different cortical areas could affect monkeys' eye movement and influence decision making. ${ }^{6-10}$ Recently, it has been reported that monkeys can detect optogenetic stimulation at the somatosensory cortex. ${ }^{12}$ These studies demonstrated not only the power of optogenetics to investigate brain function, but also the possibility of future application in clinical settings.

Because optogenetics offers advantages over traditional electrical microstimulation or pharmacological methods in both spatial and temporal precision as well as allowing bidirectional neural manipulation (excitation versus inhibition), many

*Address all correspondence to: David L. Sheinberg, E-mail: david_sheinberg@ brown.edu nonhuman primate labs are now attempting to incorporate this novel technique in their experiments. Despite some reported successes by a handful of groups, ${ }^{4-11,13}$ many researchers still find it difficult to reliably transduce cells in the monkey brain and subsequently read out neural responses to light stimulation. Here, we illustrate step-by-step methods that we have used in our previous studies ${ }^{10,12}$ to guide others in setting up their own optogenetic experiments. The methods described here are simple, highly replicable, and all devices and materials are easy to obtain. In the following text, we describe our general procedures, including the optimization of various parameters, as well as the means of detecting modulation and verifying expression.

\section{Materials and Methods}

\subsection{General Procedures}

The objective of optogenetics is to activate (or inhibit) certain types of neurons by light with cell-type specificity. To do this, neurons need to be transduced with genes to enable the expression of certain light-activated membrane proteins. The current state-of-the-art in primates is to embed such genes into a viral construct. Therefore, a single optogenetic experiment in primates includes, as elaborated next, these five steps: virus preparation, virus testing, virus injection, stimulation/recording, and histological verification. Many variations exist between the procedures applied by different groups in these steps, especially in injection and stimulation. The following is our protocol. 


\subsubsection{Virus preparation}

A vast variety of viruses for optogenetics are commercially available from vector core facilities (i.e., the UNC Vector Core ${ }^{14}$; or Penn Vector Core $\left.{ }^{15}\right)$. In general, there are three types of opsins: excitatory (i.e., ChR2, C1V1), inhibitory (i.e., NpHR, ArchT), and step-function opsins. Each opsin is sensitive to a different wavelength of light and with different response properties upon light illumination. For reviews that compare different opsins, please refer to Yizhar et al., ${ }^{16}$ Fenno et al., ${ }^{17}$ and Mattis et al. ${ }^{18}$ In most of our experiments with nonhuman primates, we have used the viral construct AAV5-CaMKII $\alpha$-C1V1 (E122T/E162T)TS-EYFP. C1V1 is a red-shifted excitation variant of ChR2 that peaks at a wavelength of approximately $540 \mathrm{~nm} \cdot{ }^{19,20}$ This viral construct mainly targets excitatory neurons (determined by the CaMKII $\alpha$ promoter and AAV5 virus), with which reliable neural and behavioral modulation has been previously reported. ${ }^{10,12,21}$ However, the methods we detail next are applicable to all types of viruses used in optogenetics studies.

The handling of the viruses from their arrival from the vector core to the beginning of injections should be performed in the following way. The virus typically arrives from the vector core in a $100 \mu \mathrm{L}$ vial, which we immediately aliquot into smaller amounts (10 or $20 \mu \mathrm{L}$, depending on the amount we plan to use at one time plus some surplus). Aliquoting the original vial only once is critical, as multiple freeze-thaw cycles could potentially damage the virus and impair the efficacy of future injections.

\subsubsection{Virus performance verification}

In our experience, there is a significant variation in the performance of each batch of virus obtained from a vector core facility. Some batches lead to no or weak efficacy in opsin expression. Therefore, it is important to verify the performance of a viral construct before injecting it into a primate. Our approach for verification is to inject the virus into at least two rats to confirm the efficacy of opsin expression with neuromodulation experiments and histology. We have found that if a virus shows good expression in rats, it has a high chance of success in primates. While this might not be true for every viral construct (note that we have only explored a few viral constructs in our studies), we believe that if a virus does not show good expression in rats, it will likely have problems in the monkeys. Therefore, we recommend checking the viruses in rats if possible. We note that the differences in performance between different batches of viruses are likely to be related to the titer of the virus, although we have not systematically tested this possibility. In our own experiments, we have successfully used titers of both $2.0 \times 10^{12}$ and $3.0 \times 10^{12}$ molecules $/ \mathrm{mL}$.

\subsubsection{Virus injection}

Injections can be performed either in the operating room (OR) while the monkey is anesthetized ${ }^{5,9}$ or in the primate chair while the monkey is seated under head restraint. ${ }^{4,10}$ The latter approach has obvious advantages. First, chair injections are more flexible than injections in the OR, where additional veterinary personnel are needed. Second, it is also better for the animal, especially when the procedure requires multiple days to complete, as injections can be performed in the same conditions as recording sessions, without requiring stereotaxic placement, anesthesia, or sedation. These "in-chair" injections are, of course, only feasible when there is a pre-existing chamber or accessible burr hole in the skull, providing access to the brain. However, the "in-chair" injections require rigid animal restraint and calming animals; movement needs to be kept to a minimum to avoid potential tissue damage (also see Discussion).

Injection equipment used by different groups differs significantly. Typically, an injection needle and a micropump are used. Additionally, other equipment to position the injection needle at the precise target location is required. Both Han et al. ${ }^{4}$ and Diester et al. ${ }^{5}$ injected viruses through a needle which was connected to a syringe via an oil-filled polyimide-coated glass tube. We found that this method was not optimal for controlling the injection volume as the dead space between the syringe and the needle potentially results in a significant amount of virus wasted in the tube. Therefore, it is preferable to shorten the path and directly connect the needle with the syringe. Cavanaugh et al. ${ }^{8}$ performed the injection using a custom-built injectrode, which enabled them to inject immediately after identifying a target site. The main limitation of their injectrode is its size, which creates unavoidable tissue damage and makes it unsuitable for repeated use.

As an improvement over the existing paradigms, we developed our injection system as illustrated in Fig. 1, which could easily be applied in-chair with reduced tissue damage. Using this system, we control the injection needle as we normally control a recording electrode, and perform the injection while the animal is head-fixed as in a recording session. We modified our primate chair to hold a stereotaxic rail on which injection equipment could be rigidly held. The same setup could be adapted for OR injections by mounting it on the surgery stereotax. The virus was withdrawn into a $25-\mu \mathrm{L}$ syringe cemented to a 32 -gauge needle (Model 702 SN, Hamilton, Reno, Nevada). The syringe was mounted on a microsyringe pump (UltraMicroPumps III, World Precision Instruments, Sarasota, Florida), which was used to control the injection volume and speed by directly

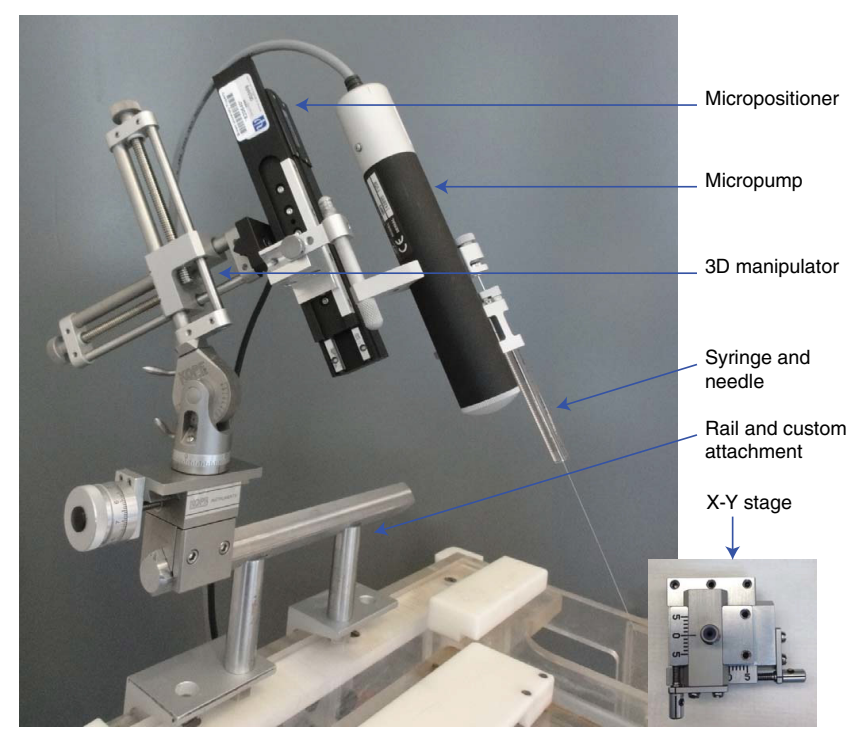

Fig. 1 Injection equipment and organization. A cemented syringe and needle are mounted on a micropump. A micropositioner and a threedimensional (3-D) manipulator are used to control the positioning of the needle. A standard Kopf rail is attached to the chair and serves as a rigid base. The inset on the bottom right shows the Kopf $X-Y$ stage, which is used to locate the position in the chamber. 
controlling the movements of the plunger of the syringe. A micropositioner (In vivo manipulator, single axis, Scientifica, East Sussex, United Kingdom) holding the pump via a custom built piece (silver block attached to the micropositioner in Fig. 1) was mounted on the stereotaxic apparatus [Kopf, threedimensional (3-D) manipulator] to accurately locate the target depth (with micrometer resolution), and the 3-D manipulator was used to adjust the rough position and angle. While targeting a particular location in the brain is straightforward with our system, before injection it is important to verify that the target brain site for injections is relevant for the particular study. To verify this, we recommend performing electrophysiological recordings beforehand to map the region functionally.

In many cases, the target region covers a significant volume in the brain. Based on histology, we have found our injections routinely lead to opsin expression in a cylindrical brain volume of a 1 to 2 -mm diameter and $\sim 1 \mathrm{~mm}$ height. Therefore, in cases where we would like to transduce neurons in a large volume, we inject the virus at different depths spaced $1 \mathrm{~mm}$ apart at each penetration site. The procedure for multidepth injections is as follows. Once the needle had been aligned with the guide tube (25 gauge), we lower the needle to the deepest target location (typically 8 to $9 \mathrm{~mm}$ in the lateral intraparietal area (LIP)). We start the injection from the deepest site, and then retract to a shallower site $1 \mathrm{~mm}$ above it. The reasons for starting with the deepest site are, first, not to interfere with sites that have already been injected. Passing the needle through these sites could indeed affect the diffusion of the viral solution and lead to adverse effects. Second, starting from the deepest site allows for deposition in the penetration track with subsequent diffusion and avoids potential reflux. Typically, one microliter (although larger volumes of $2 \mu \mathrm{L}$ have also been used by our group) of virus per depth location is injected at a speed of 100 to $200 \mathrm{~nL} / \mathrm{min}$. A waiting time of $5 \mathrm{~min}$ after each injection seems to allow diffusion before retracting the needle. Usually, to obtain a larger transduced area, injections at multiple penetration sites (separated $\sim 1 \mathrm{~mm}$ from each other on the cortical surface) and at multiple depths are recommended.

\subsubsection{Optical stimulation and neural recording}

A typical optical stimulation system includes a light source, delivery path and output, and control by an external TTL/analog signal. In all published optogenetic studies in primates, a laser coupled to an optical fiber has been utilized as the light source. This configuration can provide sufficient optical power [lightemitting diodes (LEDs) can be another option, but keep in mind that the LED wavelength spectrum is broader and coupling efficiency to an optical fiber is low). Lasers with fiber-optic couplers are commercially available (e.g., OptoEngine LLC, Coherent Inc., Omicron Laserage, and so on). An optical fiber connected to the coupler can deliver light into the monkey brain. To avoid excessive tissue damage, a small outer diameter is recommended for a fiber that needs to go into the brain. Previous studies mainly used fiber of 200 to $250 \mu$ m diameter. ${ }^{4-6,8}$ The size of the fiber includes the core, the cladding, and the coating. The coating provides extra buffer layer to protect the fiber (i.e., the fiber mentioned previously has a coating diameter of about $500 \mu \mathrm{m}$ ), and usually it will be removed before use. Note that different fibers having the same core size may have different cladding size. Caution should be used when indicating fiber size in publications. In our previous papers, ${ }^{10,21}$ smaller fibers with $125-\mu \mathrm{m}$ cladding (10 or $50 \mu \mathrm{m}$ core) were used.
Another important parameter of the optic fiber is the numerical aperture (NA), which is a dimensionless number representing the range of angles at which the fiber can accept or emit light. In general terms, a larger NA means a broader dispersion of light emitted from the fiber.

One issue reported by previous studies is that fibers with blunt tips can cause tissue damage and subsequently make longterm recording difficult. ${ }^{6,22}$ To address this, we taper the fiber to a tip angle of approximately $10 \mathrm{deg}$ [ $~ 8 \mathrm{deg}$ in Fig. 2(b)] using a laser puller (P-2000, Sutter Instruments). This modification, together with using a smaller-diameter fiber, significantly reduces tissue damage and renders it possible to repeatedly penetrate the same area for months (more than 6 months; generally, more than 100 penetrations were done for each monkey). Another benefit of such modification is that a significant amount of light can exit the fiber along the edges of the taper before reaching the tip (Fig. 2), which increases the volume of tissue illuminated compared to a blunt tip, and thereby reduces the peak light intensity in the tissue for a given power. In general, special attention should be given to the amplitude of peak light intensity in the tissue since high light intensity could cause tissue damage or nonspecific heat-induced effects. ${ }^{21}$

Different stimulation parameters, including power, frequency, and duration, were applied by previous studies. In these studies, either "power" or "intensity" was used to report the amount of illumination, but these are different physical quantities which use completely different units. Power is the energy emitted per unit time, in units of watt (W), milliwatt (mW), and so on, which can be directly measured using a power meter. Intensity is the power per unit area, in units of $\mathrm{W} / \mathrm{m}^{2}$. One can easily measure the power of a laser by placing the output fiber into the sensor of a power meter, but further calculation is needed to determine intensity. For example, the light intensity at a blunt fiber end is the optical power divided by the area of the fiber core, but for a tapered fiber, the core is attenuated after pulling; therefore, the intensity will vary along the tip. In brain tissue, the light will scatter after exiting the fiber and the intensity will dramatically drop at increasing distances. To see a simulation of light intensity distribution in brain tissue, please refer to Ozden et al. for a blunt fiber ${ }^{21}$ and Dai et al. for a tapered fiber. $^{10}$

In terms of optogenetic stimulation, it is of great interest which illumination parameters are optimal. Unfortunately, there is no clear-cut answer for this as these parameters depend on the fiber, opsin, brain area, and other factors. Before setting these parameters, one has to be careful about the heat accumulation around the fiber tip due to light absorption by the tissue, which potentially could damage the brain. To ensure safety, we have previously calculated that, given certain assumptions and with continuous light illumination, the power should not exceed $12 \mathrm{~mW}$ for a $10-\mu \mathrm{m}$ core fiber and $40 \mathrm{~mW}$ for a $200-\mu \mathrm{m}$ core fiber. ${ }^{21}$ Note that higher powers can be applied when pulsed (instead of continuous) stimulation is used, depending on the stimulation duty cycle. However, with our setup we have found that a few hundred microwatts is sufficient to activate neural activity when using the C1V1(T/T) opsin.

One advantage of optogenetics is that it allows simultaneous recording during stimulation. Several special readout devices, namely optrodes, have been designed for this purpose. ${ }^{21,23,24}$ Lacking these more advanced and expensive tools, we have found an alternative solution is to build an optrode by gluing an electrode and a fiber together. Note that the optrode used in 
(a)

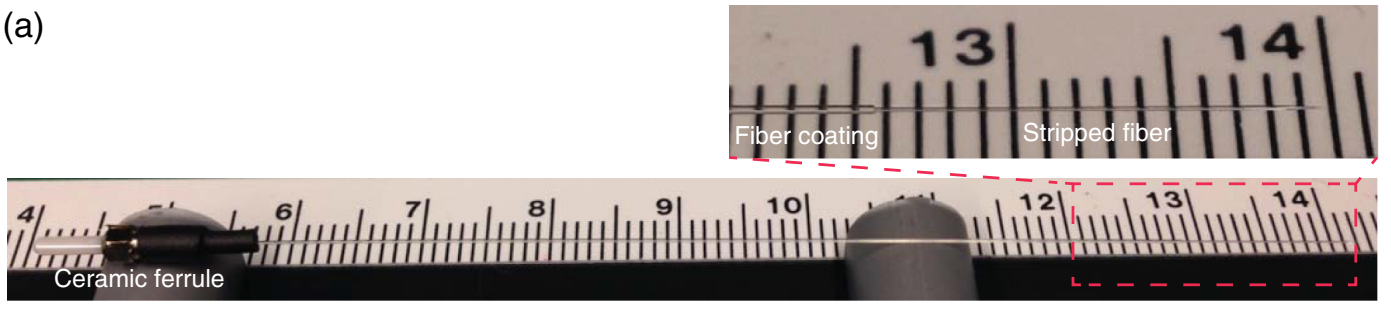

(b)

(c)
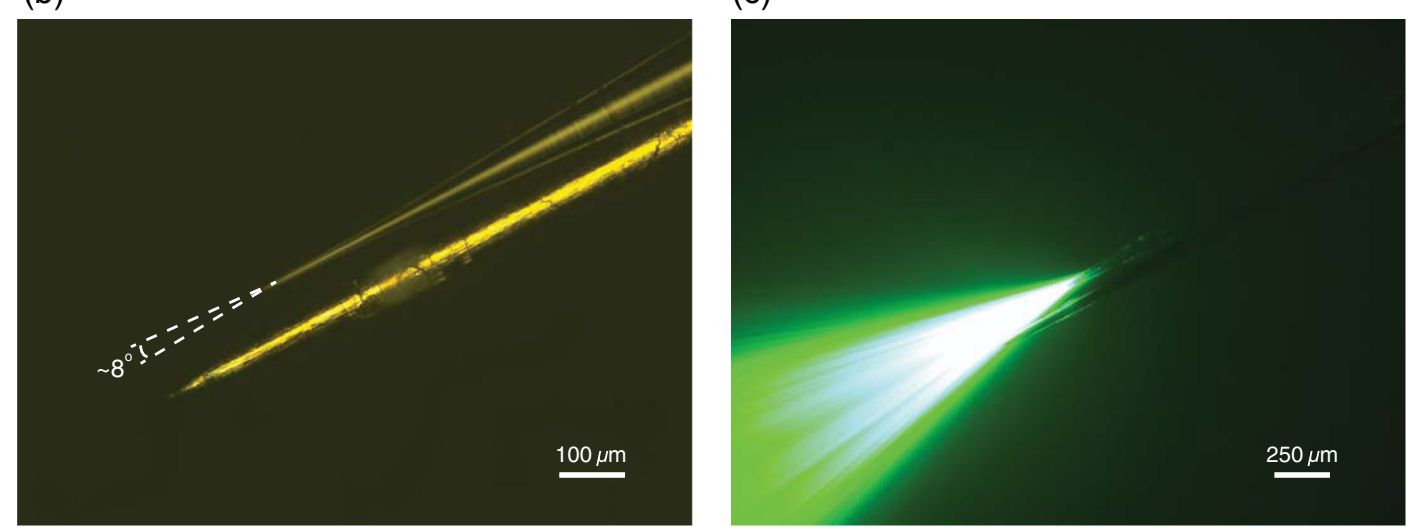

Fig. 2 Tapered fiber and optrode. (a) A pulled fiber after connecting to a ferrule and polishing of the ceramic end. The inset zooms in the tapered end, which also shows the fiber with and without coating. (b) The tip of the optrode under high-resolution microscope, which shows the relative position of the tapered fiber and the tungsten electrode. (c) Illumination of light scattering from the fiber tip.

Diester et al. ${ }^{5}$ was a $250-\mu \mathrm{m}$ electrode glued with a $225-\mu \mathrm{m}$ fiber, so the overall size was at least $475 \mu \mathrm{m}$, which is not ideal for an acute recording device. We refined this optrode by using a smaller fiber (cladding $=125 \mu \mathrm{m}$ ) and a smaller electrode (shank diameter $=75 \mu \mathrm{m}$ ), rendering the optrode diameter significantly smaller, around $200 \mu \mathrm{m}$ in total. ${ }^{10}$ This smaller size is not dramatically larger than a standard recording electrode. Using this kind of device, we have been able to repeatedly obtain effective neural modulation from a transduced area approximately $2 \times 2 \times 4 \mathrm{~mm}$ for months without having the problem of serious cortical damage reported by Gerits et al. $^{6}$ More importantly, virtually no artifacts were observed at the single/multiunit level using our filter setting (300 to $6000 \mathrm{~Hz}$ ). Given that others have observed light-induced
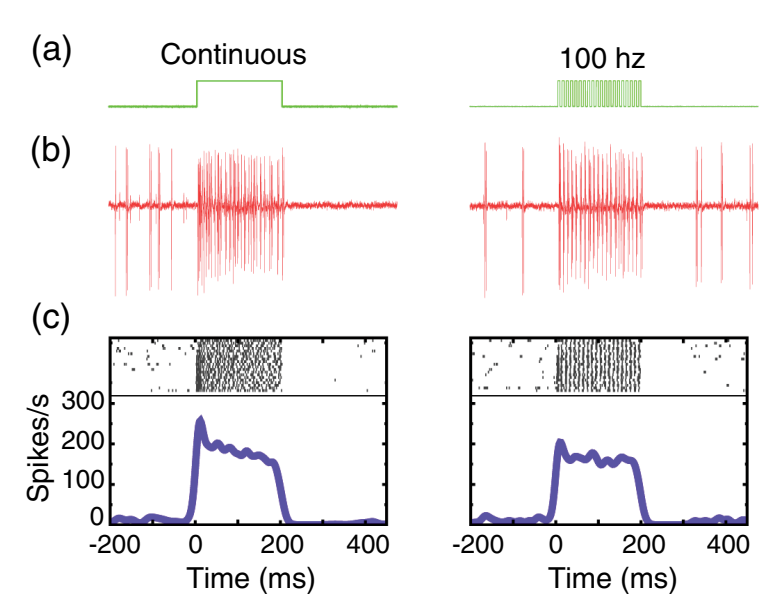

artifacts during recordings with metal electrodes and that same type of electrode was used in a similar optrode configuration, ${ }^{4}$ we think using a smaller size electrode is essential as decreasing the area of light exposure is effective in eliminating photoelectric reaction $(75 \mu \mathrm{m}$ here versus $200 \mu \mathrm{m}$ by Han et al. ${ }^{4}$ ).

\subsubsection{Expression verification}

To verify that the injected virus does indeed transduce neurons in regions of interest, the most straightforward approach is to find optically induced neural modulation in those injected sites. At sites with sufficient opsin expression, neurons should be either activated (for excitatory opsins, Figs. 3 to 4 ) or
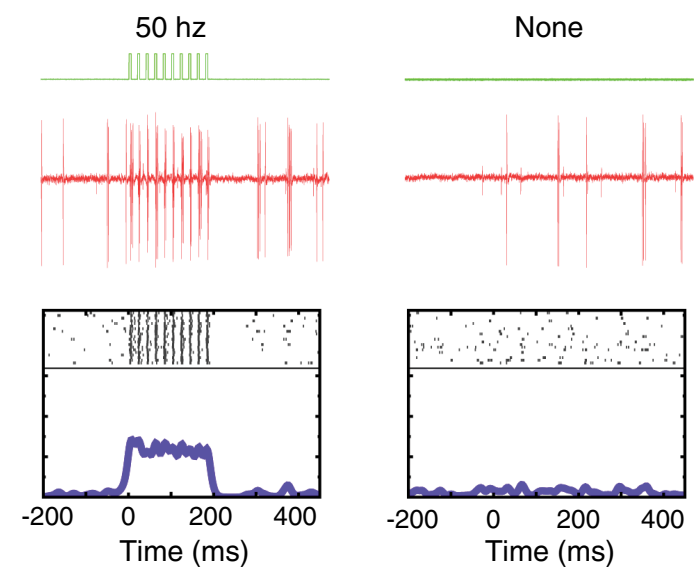

Fig. 3 Example of optogenetic modulation. Each column represents a different stimulation frequency. From top to bottom, we show: (a) a schematic of the pulse train delivered to the laser at each stimulation frequency, (b) the raw spike train from one randomly picked trial for each frequency, and (c) the raster and the spike density functions. 
(a)

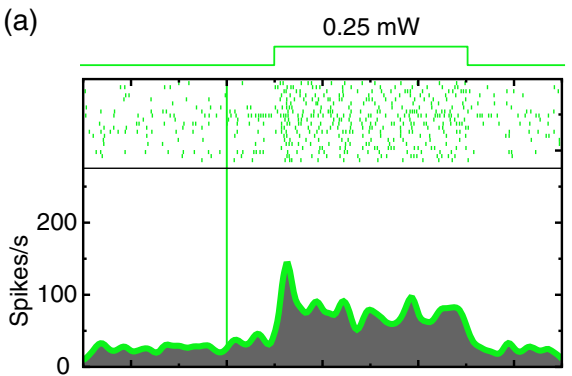

(c)

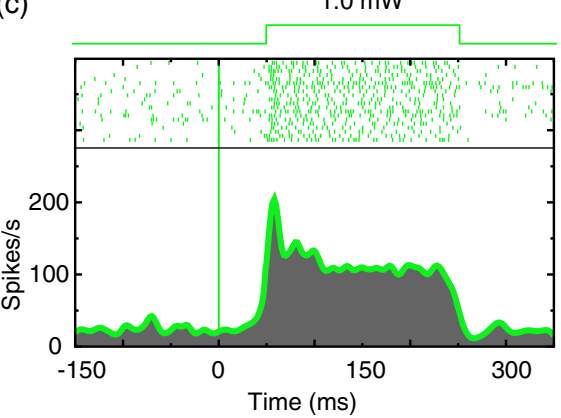

(b)

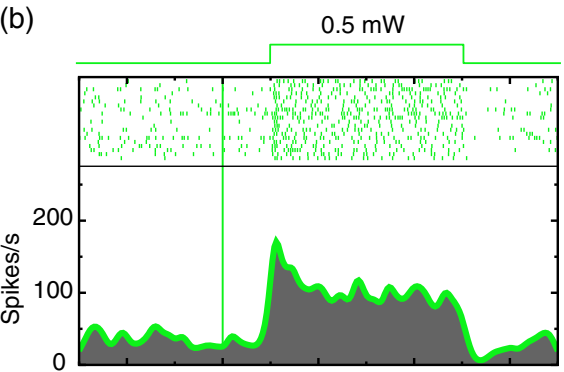

(d)

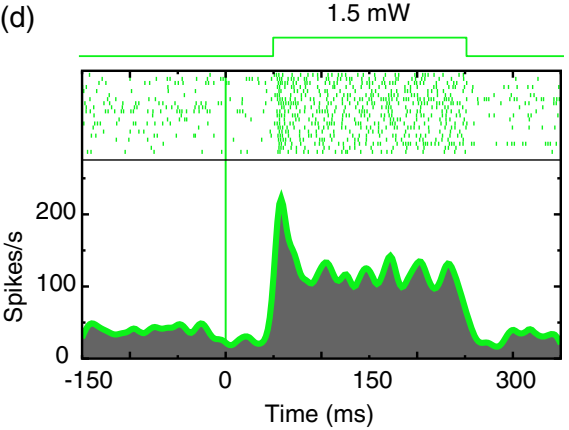

Fig. 4 Comparison of different optical stimulation powers. Neural responses to a 200 ms continuous stimulation with measured stimulation power of (a) 0.25 , (b) 0.5 , (c) 1.0 , and (d) $1.5 \mathrm{~mW}$.

suppressed (for inhibitory opsins) upon light stimulation. The question is how to find optical modulation after injection. For us, the general procedure is described as follows. We usually start by listening (using an audio monitor) to the change of background noise as we deliver light pulses through the optrode while being lowered in the brain. Typically, as the optrode gets closer to the transduced area, we begin to hear a weak hash, like the distant crash of waves, synchronized with light pulses. The sound gets stronger as the optrode gets deeper. There might be no visible spiking on the oscilloscope at this point, but by lowering the speed of progression, and listening patiently to the audio signal, eventually single/multiunit modulation can be detected in most cases after hearing this initial low-frequency noise. In our experience, the approach described previously is very efficient for identifying regions of opsin expression and finding spiking units responsive to light. As an alternative approach, one might first start looking for spiking units without checking the light responsiveness in the tissue, and then checking whether the isolated units are light-sensitive. In our hands, this approach was less efficient in finding lightresponsive units, since the isolated units could be in a region of weak or no expression. Overall, the background noise during light pulses may reflect the network effect of multiunit response, thus it should be an effective source to identify optically modulated sites.

In addition to multiunit modulation, previous studies have also reported the modulation of local field potentials (LFPs), ${ }^{4,5,9}$ Typically, for sites expressing opsin C1V1(T/T), we have observed a negative deflection in LFPs upon green light illumination, followed by a rebound after stimulation [Fig. 5(a)]. However, as reported by Han et al., such a modulation pattern can also be observed in saline as artifacts, especially when a large-size fiber and electrode are used. ${ }^{4}$ Therefore, even in a region of opsin expression in the brain, it is not straightforward to distinguish opsin-induced LFPs from light-induced artifacts. Therefore, LFP-like signals in response to light pulses do not necessarily mean that the signal is neural or that the opsin is being properly expressed in nearby tissue. In fact, light-induced artifacts are more pronounced in the LFP bandwidth and depend on both the stimulation power and wavelength of light. Even in cases where the amplitude and shape of the artifact could be potentially different from optically induced LFPs [Fig. 5(b)], we still think LFPs cannot be reliably distinguished from artifacts and therefore are not a reliable way to identify regions of opsin expression. A more meaningful way may be to first characterize the artifacts at fresh sites that have not been injected and far away from injection sites. No artifacts observed at fresh sites could increase the reliability of LFPs observed in transduced sites [Fig. 5(c)]. However, given the variety of LFP patterns for different opsins, ${ }^{5,9}$ the mechanism underlying optical-induced LFP is still unclear. The question of using LFP as a verification measurement remains open. We do recommend testing LFP artifacts in saline and in the brain beforehand.

As an alternative approach, in vivo fluorescence detection systems were proposed which allowed monitoring expression in vivo after injection. ${ }^{5,21}$ Diester et al. reported that the fluorescence measurements correlated with neuron responses to light stimulation. Theoretically, this could be an ideal substitution for histology, especially for an ongoing experiment. However, our experience has shown that detecting an increase in fluorescence in a given location does not necessarily mean optically induced neuromodulation will be present.

The ultimate verification consists of histology, which is reliable and useful to characterize the efficacy of expression. However, we do not recommend sacrificing every individual subject for this purpose. Instead, we suggest testing the expression efficacy in a rat as we have observed very similar result in both recording and histology.

\subsubsection{Validation of the functionality of viral construct in rats}

As previously mentioned, performing virus injections in rats is suitable for validation of virus efficacy and can be used in lieu of 
(a)

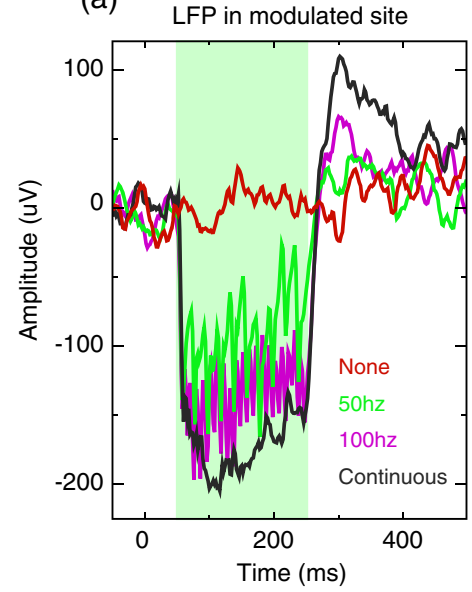

(b)

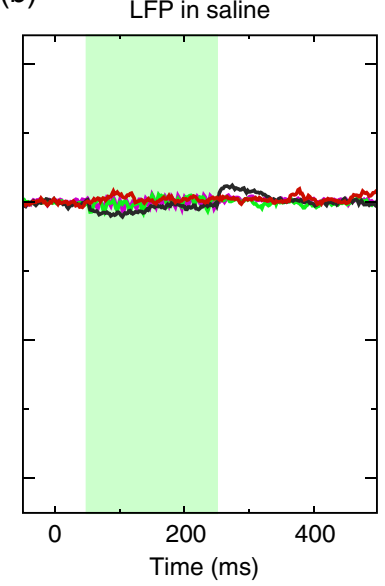

(c) LFP in non-transduced cortex

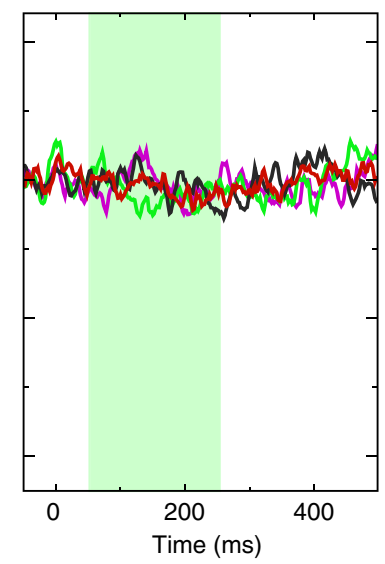

Fig. 5 Typical local field potential (LFP) versus artifact. (a) Typical LFP recorded in a site transduced with C1V1(T/T). Black, purple, and green represent stimulation of continuous, 100 and $50 \mathrm{~Hz}$, respectively. Red is the base line when no stimulation was applied. Stimulation period is indicated by the green shadow. Same conventions are used for b-c. (b) LFP recorded in saline. (c) LFP recorded in a nontransduced site.

primate histology. Our protocol is detailed next. The viral construct to be tested was injected into two cortical areas (usually motor, somatosensory, or posterior parietal cortical areas) from the same hemisphere in two rats. As a control, a viral construct already tested and of known efficiency was injected into the same cortical areas on the other hemisphere. The injections were performed as follows: rats were mounted on a stereotaxic frame (Model 1730, Kopf Instruments) under isoflurane (2\%) anesthesia, and under aseptic conditions, a small skin incision $(\sim 10 \mathrm{~mm})$ was made to expose the skull above the target areas for injections. Small burr holes $(\sim<1 \mathrm{~mm})$ were made at each site to expose the brain with the dura intact. Through each burr holes, the virus was injected at two depths $(0.5$ and $1.5 \mathrm{~mm})$ with the same micropump-syringe system described previously mounted to a stereotaxic micromanipulator. The injection amount and speed were similar to that used in primates, i.e., $1 \mu \mathrm{L}$ of virus per depth at a speed of $100 \mathrm{~nL} / \min$ and $5 \mathrm{~min}$ waiting time after injections. After injections were completed, the burr holes were covered with bone wax and the skin was sutured. After allowing at least 3 weeks for opsin expression, the rats were anesthetized with either isoflurane $(\sim 2 \%)$ or ketamine/xylazine $(10 \mathrm{mg} / \mathrm{kg}$ ) and the sites injected with the investigated virus were exposed with two craniotomies $(\sim 2 \times 2 \mathrm{~mm})$. The location of viral expression was first determined by investigating the yellow fluorescent protein (YFP) fluorescence (YFP is coexpressed with the opsin) at the brain surface. For this purpose, we delivered 473-nm laser light over the craniotomy and observed the YFP fluorescence with a stereomicroscope carrying a YFP filter. Usually, YFP fluorescence signal was visible at the surface throughout the craniotomy. After verifying the expression and finding the site of peak expression by observing the fluorescence, we validated neuromodulation by inserting an optrode into the region of peak fluorescence intensity and delivering occasional light pulses $(\sim<1 \mathrm{~mW}, 561 \mathrm{~nm})$ of 1 to $2 \mathrm{~s}$ duration and observing single and multiunit spiking activity. Once the functional expression was validated, the rats were perfused with $2 \%$ paraformaldehyde to prepare $60 \mu \mathrm{m}$ thick histological slices. The slices were investigated for the extent of YFP expression under a fluorescence microscope. This time, the YFP fluorescence intensities and pattern of expressions due to injections of the tested and control viruses were compared. If they appeared to be similar, we concluded that the new viral construct was suitable for primate use.

\subsection{Materials}

\subsubsection{Reagents}

Virus: AAV5-CaMKII $\alpha$-C1V1(E122T/E162T)-TS-EYFP(UNC vector core);

Dilute bleach (10\% dilute bleach);

95\% ethanol (Fisher Science Education, cat. ID (catalog ID): A405F-1GAL);

Cidex solution (Advanced Sterilization Products CIDEXPLUS 28 day solution, cat. ID: 2683/2785);

Chlorhexidine solution (Vet Solution, cat. ID: 91010);

Sterile saline.

\subsubsection{Equipment}

Virus aliquot

PCR tube (TempAssure $0.5 \mathrm{~mL}$, available at usascientific.com, cat. ID: 1405-8108);

Filter tip (TipOne 0.5 to $10 / 20 \mu \mathrm{L}$, available at usascientific.com, cat. ID: 1121-4810);

Pipette (ErgoOne 2 to $20 \mu \mathrm{L}$ single channel, available at usascientific.com, cat. ID: 7100-0220).

\section{Virus injection}

Cemented syringe and needle (Hamilton, $25 \mu \mathrm{L}$, 32 gauge, custom length, point type 4, cat. ID: 80408);

Micropump (WPI, UltraMicroPump with SYS-Micro4 controller, cat. ID: UMP3-2);

Micropositioner (Scientifica, in vivo manipulator, single axis, cat. ID: IVM 1000);

Stereotaxic arm (Kopf, Model 1460 three-dimensional manipulator);

Stereotaxic rail (Kopf, Model 1530 Frame); 
Custom rail attachment (custom piece that could attach the rail to the chair);

PCR tube holder (custom piece that could hold the tube and make bottom of the tube visible);

Guide tube (25 gauge, custom length);

$\mathrm{X}-\mathrm{Y}$ stage (Kopf, Model 608-B X/Y slide).

\section{Laser delivery}

561-nm laser (OptoEngine, 561-nm laser, MGL-FN-561/ $100 \mathrm{~mW}$ with TTL/analog modulation);

Laser adapter (OptoEngine, fiber coupling adapter with SMA905 connector);

Fiber patch cord (Thorlabs, Custom patch cord, fiber cat. ID: FG050LGA, End A: SMA905, End B: $1.25 \mathrm{~mm} \mathrm{LC} \mathrm{ceramic}$ ferrule, Jacket: FT030);

Pulse generator (custom program that can send TTL/analog pulse of different frequency and duration).

\section{Optrode fabrication}

Optical fiber (Thorlabs, $50 \mu \mathrm{m}$ core, $125 \mu \mathrm{m}$ cladding, cat. ID: FG050LGA);

Fiber connector (Precision Fiber Products, LC $1.25 \mathrm{~mm}$ OD multimode ceramic zirconia ferrule, cat. ID: MMFER2007CF-1270);

Sleeve (Precision Fiber Products, ceramic split sleeve, cat. ID: SM-CS125S);

Fiber stripping tool (Thorlabs, cat. ID: T06S13, Cladding/ Coating size: $125 / 250 \mu \mathrm{m})$;

Laser puller (Sutter Instruments, Laser-based micropipette puller, cat. ID: P-2000/F);

Epoxy (Thorlabs, Epoxy for fiber optic connectors, cat. ID: F123);

Electrode (FHC, $125 \mu \mathrm{m}$ tungsten electrode, cat. ID: UEWLCESM7N4G);

Hot plate (Amazon, Corning Digital Hot Plate, $5^{\prime \prime} \times 7^{\prime \prime}$, 120 VAC);

Heat shrinking tube (DigiKey, Heat Shrinking tubing kit, cat. ID: Q2Z1-KIT-ND);

Polishing disk (Precision Fiber Products, aluminum fiber optic polishing disk for LC connectors, cat. ID: M1-80754);

Polishing plate (Thorlabs, glass polishing plate, cat. ID: CTG913);

Polishing sheet (Thorlabs, $5 \mu \mathrm{m}$ grit polishing sheet, cat. ID: LFG5P);

Polishing sheet (Thorlabs, $1 \mu \mathrm{m}$ grit polishing sheet, cat. ID: LFG1P);

Microscope (Zeiss, Model Axiostar);

Super glue (Krazy, all-purpose glue pen);

Silicone tube (A-M Systems, biomedical silicone tubing, cat. ID: 806400);

Power meter (Thorlabs, compact power and energy meter console, cat. ID: PM100D);

Power sensor (Thorlabs, Integrating sphere photodiode power sensor, cat. ID: S140C).

\section{Recording}

Electrode holder (Kopf, Model 608 chronic adapter);

Micropositioner (Kopf, Model 2650, hydraulic with $25 \mathrm{~mm}$ travel);

Headstage (TDT, ZC16, 16-channel ZIF-Clip headstage);

Amplifier (TDT, PZ2-32, 32-channel preamplifier);

Data acquisition system (TDT, RZ2 BioAmp Processor).

\subsection{Experimental Procedures}

\subsubsection{Aliquoting virus}

The purpose here is to aliquot the virus into individual $20 \mu \mathrm{L}$ vials.

1. First, thaw the virus vial (usually $100 \mu \mathrm{L}$ ) sent from vector core in ice for $30 \mathrm{~min}$.

2. Place the virus vial, five PCR tubes (optimally sterilized under UV light for $20 \mathrm{~min}$ before hand), and filter tips, as well as $10 \%$ dilute bleach in the fume hood. Set the pipette to withdraw $20 \mu \mathrm{L}$.

3. Use the pipette to tap the filter tip in, withdraw $20 \mu \mathrm{L}$ virus then infuse into one PCR tube. Discard the filter tip into bleach.

4. Repeat step 3 to aliquot the $100 \mu \mathrm{L}$ into a total of five tubes.

5. Label each tube with virus name, amount, and batch number.

\subsubsection{Injecting virus}

The injection plan here is to inject 2 to 3 locations covering 5 to $6 \mathrm{~mm}$ depth each.

6. Before injections, measure the length of the needle. Sterilize the needle by soaking the syringe in cidex solution for $2 \mathrm{~h}$. Remember to sterilize the inside of the needle by withdrawing cidex into the syringe. Then flush the needle and syringe with sterile saline (both inside and out), followed by flushing with ethanol (as ethanol evaporates more quickly). Separate the syringe and plunger and place them on a sterile pad to let them air dry. Another option is to sterilize the needle and syringe using ethylene oxide.

7. Setup the stereotaxic arm, the micropositioner (including the software and interface), and the micropump and mount them together to standby. Mount a dummy syringe onto the pump, which will help the alignment in step 12 .

8. Measure the length of the guide tube. Sterilize the guide tube and put it on the $\mathrm{X}-\mathrm{Y}$ stage after setting the $\mathrm{x}-\mathrm{y}$ coordinate to preidentified location.

9. Take out one vial of virus $(20 \mu \mathrm{L})$ from freezer and thaw it in ice.

10. Seat the monkey with head restraint. Mount the rail onto the monkey chair.

11. Clean the chamber and put on the stage. Penetrate the dura with guide tube and fix it in place.

12. Mount the stereotaxic arm and other equipment on the rail. Adjust the angle and position of the 3-D manipulator to align the dummy needle with guide tube. This usually takes some time to get a close alignment. Taking note of the readings on the 3-D manipulator will help when repositioning the manipulator at this same location in step 17 . 
13. Replace the dummy needle with the sterile needle.

14. Take out the virus from ice after it has thawed and put the PCR tube on the tube holder. Adjust the 3-D manipulator to align the needle with the tube. Lower the needle into the virus using the interface of the Scientifica micropositioner, which gives much more stable movement than the Kopf manipulator. Make sure that the needle can reach the bottom of the tube without touching the wall. To view the needle's position inside the PCR tube, it can often help to point a light source directly at the tube during this step.

15. Connect the micropump to the controller (channel 1). Set channel 1 to $\mathrm{W}$ (withdraw), set volume to $15,000 \mathrm{~nL}$, set speed to $1000 \mathrm{~nL} / \mathrm{min}$, set syringe type to $\mathrm{E}$ (corresponding to $25 \mu \mathrm{L}$ ). Press the start button to start withdrawing. Note that there will be about $1.25 \mu \mathrm{L}$ dead space in the syringe (this depends on the needle and syringe). Monitor the needle carefully and make sure the tip is in the virus all the time.

16. After withdrawal is done, check the withdrawn volume inside the needle, and then retract the needle. Make sure there are no bubbles inside the syringe. Discard the virus left in the PCR tube into the dilute bleach solution. Alternatively, freeze the remaining virus again for reuse.

17. Align the needle back to the guide tube using the measurements obtained in step 12. Lower the needle through the guide tube using the manipulator or the micropositioner. Measure the length of the needle left over the guide tube while lowering; make sure it does not pass through the other end (minimum left over length=needle length-guide tube length).

18. After the needle reaches the end of the guide tube, lower the needle at low speed $(10 \mu \mathrm{m} / \mathrm{sec})$ using the Scientifica software to reach the deepest target depth. Check the remaining length of the needle to verify that the needle has reached the target position.

19. Set the micropump controller to I (Infuse), set the volume to $1000 \mathrm{~nL}$, set speed to $200 \mathrm{~nL} / \mathrm{min}$. Press start button to start injection.

20. After the injection is done, wait another $5 \mathrm{~min}$.

21. Retract the needle for $1 \mathrm{~mm}$ at low speed $(5 \mu \mathrm{m} / \mathrm{s})$.

22. Repeat steps 19-21 until all desired depths have been infused.

23. Retract the needle and move the guide tube to another preidentified trajectory. Repeat steps $17-22$ to inject another trajectory.

\subsubsection{Making the optrode}

The purpose here is to make a tapered fiber of $\sim 10 \mathrm{~cm}$ length and then glue with a tungsten electrode of approximately the same length. Readers are welcome to contact the authors to obtain further instruction.

24. Cut the optic fiber to about $13 \mathrm{~cm}$ length.
25. Strip the coating using the stripping tool at one end and expose the cladding for about $4.5 \mathrm{~cm}$.

26. Place the fiber piece into the laser puller and fix both ends. Make sure the coating on the burn point has been removed.

27. Allow the pipette puller to warm-up for at least $15 \mathrm{~min}$ before use. Set the parameter on the puller as: $\mathrm{HEAT}=305, \mathrm{FIL}=$ Empty, $\mathrm{VEL}=20, \mathrm{DEL}=$ 126 , and PUL $=150$. The setting could be different for a different machine and different fiber. One should find out the optimal setting to obtain an ideal taper shape for one specific type of fiber. Press start to pull. It should complete in 1 to $2 \mathrm{~s}$.

28. Take out the pulled fiber and check the length of the exposed cladding. Normally, about $1 \mathrm{~cm}$ is required. Check the tip shape under microscope.

29. Remove the coating of the other blunt end of the fiber for about $1 \mathrm{~cm}$. Apply some epoxy on the fiber end and put on a ceramic ferrule. Make sure the fiber passes through the ferrule.

30. Set the hot plate to $100^{\circ} \mathrm{C}$. Put the fiber and the ferrule on the plate. The epoxy will turn brown and become hard under heating.

31. Put a short piece of heat shrinking tube between the fiber and ferrule to secure the connection.

32. When the fiber is rigidly connected to the ferrule, break the exposed fiber with a forcep or scissors all the way close to the ferrule, and then put the ferrule into the center hole of the polishing disk.

33. Place the 5 and $1-\mu \mathrm{m}$ polishing sheet on the polishing plate side by side. Hold the polishing disk center and the ferrule together and start polishing on the $5-\mu \mathrm{m}$ sheet, then move to the finer $1 \mu \mathrm{m}$ one. Apply some water on the sheet while polishing. Move the polishing disk in a trajectory that is as in writing the digit 8 . Complete 20-30 cycles for each sheet.

34. Take out the fiber and check the surface of the polished end under microscope. The surface should be flat like a mirror, and the core can be seen clearly at the center. Put on a ceramic sleeve after the polishing appears satisfactory (optional).

35. Check the connection efficiency by connecting the fiber to a LC type laser output (refer to step 39). Measure the power output from the tapered fiber tip.

36. Place an electrode next to the fiber. Putting them both through a tube can render it easier to place them together. Make the electrode leads ahead of the fiber for about $200-300 \mu \mathrm{m}$.

37. Apply a small amount of super glue on the fiber and electrode from the tip to the shank to glue them together. Make sure they are not twisted but stay parallel all the way. Put on a piece of silicone tube, which will help the electrode holder to hold the optrode and protect the fiber. 


\subsubsection{Stimulating and recording}

38. Wait approximately 4 weeks after injection before exploring the expression.

39. Setup the laser system. Put the laser adapter on the laser head. Connect the fiber patch cord to the adapter. Adjust the three screws on the adapter to get a good coupling to the fiber.

40. Connect the pulse generator to the laser via BNC cable. Set the modulation model on the laser to TTL (or analog if the signal source is analog). Test the control signal using a scope and monitor the laser output.

41. Connect the optrode to the other end of the patch cord fiber. Measure the output power from the optrode tip using a power meter. Adjusting the current or voltage on the laser interface to obtain an output power around $1 \mathrm{~mW}$.

42. Load the optrode onto Kopf electrode holder and X-Y stage through a guide tube. Soak the optrode into chlorhexidine solution for half an hour (for disinfection). Then flush with ethanol.

43. Mount the stage on the recording chamber. Set the coordinate to the injected location. Penetrate the dura with the guide tube and lower the optrode manually to the guide tube bottom.

44. Lower the optrode using the Kopf micropositioner at low speed. Send a light pulse to the brain (using custom program) and listen to the audio monitor. Normally, using this kind of optrode, we do not hear any special sound when the optrode is far away from expression site. However, as the optrode gets closer, we can consistently hear some modulated hash or background noise responding to light stimulation. Keep lowering the optrode until significant change caused by light appears in the signal.

45. Record light-induced modulation or perform another behavioral task with optical stimulation.

\subsection{Timing}

Steps 1-5, aliquoting virus: $40 \mathrm{~min}$;

Steps 6-8, preparation for the injection: $3 \mathrm{~h}$;

Steps 9-23, performing the injection: $4 \mathrm{~h}$;

Steps 24-37, making optrode: $\sim 1 \mathrm{~h}$;

Step 38, waiting for virus express: 4 weeks;

Steps 39-41, preparation for the optical stimulation: Iih;

Steps 42-45, probing the expression: $2-4 \mathrm{~h}$.

\subsection{Troubleshooting}

Troubleshooting advice can be found in Table 1.

\section{Results}

\subsection{Cell Type Specific Neural Activation}

The virus we have primarily used (AAV5) with the CaMKII $\alpha$ promoter mainly targets excitatory cells. We have successfully expressed C1V1 in multiple areas (including LIP, IT, S1, and $\mathrm{PMv}$ ) in multiple monkeys. Typically, cells expressing the C1V1(T/T) opsin will be activated upon green light illumination. Figures 3(a)-3(c) show the responses of one example neuron from LIP when stimulated with $200 \mathrm{~ms}$ light pulses $(561 \mathrm{~nm}$, $1.0 \mathrm{~mW}$ ) at different frequencies (continuous, 100 and $50 \mathrm{~Hz}$ ), compared to spontaneous responses. This neuron was strongly modulated and time-locked to the light pulse. Figure 3(b) shows the raw spike train from one randomly chosen trial, from which we did not observe any artifact caused by light onset or offset. We also noticed that the amplitude of the optogenetically evoked spikes was almost identical to spontaneous spikes, even if a slight reduction was observed upon continuous or high-frequency stimulation. It was also very common to observe poststimulation inhibition, i.e., the neuron remaining quiet for $\sim 100 \mathrm{~ms}$ after the end of optical stimulation. This quiet period varied depending on the strength of stimulation. Generally speaking, higher power or higher frequency caused longer poststimulation inhibition.

To demonstrate the influence of optical power on neuronal responses, we further characterized the neural response properties to different stimulation power settings. Figure 4 shows the response of one neuron to four power levels $(0.25,0.5,1.0$, and $1.5 \mathrm{~mW}$ ). Interestingly, even at power levels as low as $0.25 \mathrm{~mW}$, this neuron could be activated. The response amplitude also seemed to saturate at a certain level, as stimulation of $1.5 \mathrm{~mW}$ did not evoke a much stronger response than $1.0 \mathrm{~mW}$ (Fig. 4). Therefore, in this case, activating a transduced cell did not require a particularly high power (only a few hundred microwatts).

\subsection{Optogenetic Local Field Potential versus Artifacts}

We have shown that the optrode we were using had no heatinduced artifact in the spiking activity. The effect on LFPs was also investigated. Typical LFPs upon optical stimulation at one transduced site are shown in Fig. 5(a), which normally shows a quick negative response after light onset, followed by a rebound after light offset. Time-locked phases can also be seen corresponding to stimulation frequency. The LFP amplitude here (upon $\sim 1.0 \mathrm{~mW}$ stimulation) is about 100 to $200 \mu \mathrm{V}$ for the negative phase, but varies according to stimulation power. When we tested the LFP in saline using the same stimulation protocol, we found some artifacts upon stimulation, but the amplitude was much smaller than true optogenetically evoked LFPs [Fig. 5(b)]. Given that the scattering property in saline could be different from that in the cortex, we further tested the artifact in nontransduced sites of the brain and found no artifact at all [Fig. 5(c)]. We believe, then, that the LFPs we observed in Fig. 5(a) have a neurophysiological origin. However, we also noted that the artifact observed in saline depended on the type and size of electrode, the optical power, and the wavelength. Therefore, testing these properties of the optrode before using it for actual experiments is strongly recommended.

\subsection{Histology Validation of Virus Expression}

We performed histological validation of viral expression in two different contexts. The first one is for validation of the functionality of the viral construct in rats before injection into primates. The second one is for histological studies of the extent of opsin 
Table 1 Troubleshooting table.

\begin{tabular}{|c|c|c|c|}
\hline Step & Problem & Possible reason & Solution \\
\hline 12 & $\begin{array}{l}\text { Unable to get a close } \\
\text { alignment }\end{array}$ & $\begin{array}{l}\text { The relative positioning of the } \\
\text { micropositioner, the micropump; the limit of } \\
\text { the 3-D manipulator }\end{array}$ & $\begin{array}{l}\text { Adjust the placement of the micropump } \\
\text { and the micropump; use other manipulator } \\
\text { arm with more dimensions of freedom (i.e., } \\
\text { Brainsight Arm) }\end{array}$ \\
\hline 14 & $\begin{array}{l}\text { Unable to see the } \\
\text { needle in the PCR tube }\end{array}$ & The bottom of the tube is blocked & $\begin{array}{l}\text { Use a thin holder that only holds the top of } \\
\text { the tube but exposes the bottom, and then } \\
\text { projects some light on the tube from the side }\end{array}$ \\
\hline 15 & $\begin{array}{l}\text { Withdraw bubble in } \\
\text { the syringe }\end{array}$ & The needle tip is out of reach to the virus & $\begin{array}{l}\text { Infuse the virus back to the tube and } \\
\text { withdraw again }\end{array}$ \\
\hline 17 & $\begin{array}{l}\text { Lost alignment after } \\
\text { lowering the needle }\end{array}$ & $\begin{array}{l}\text { The needle itself is not well-aligned with } \\
\text { the micropositioner }\end{array}$ & $\begin{array}{l}\text { Adjust the alignment based on the angle of } \\
\text { the micropositioner }\end{array}$ \\
\hline 27 & The fiber is not pulled & $\begin{array}{l}\text { The coating on the burn point has not } \\
\text { been removed; the fiber in the puller } \\
\text { track is not well aligned }\end{array}$ & $\begin{array}{l}\text { Remove enough coating; place the fiber } \\
\text { well-aligned in the track }\end{array}$ \\
\hline 28 & $\begin{array}{l}\text { The exposed part of the } \\
\text { pulled fiber is too short }\end{array}$ & $\begin{array}{l}\text { Not enough coating has been removed; } \\
\text { the placement of the fiber in the puller is } \\
\text { too close to the right side }\end{array}$ & $\begin{array}{l}\text { Remove more coating; place the fiber in the } \\
\text { track a little more left }\end{array}$ \\
\hline 35 & $\begin{array}{l}\text { No light output or low } \\
\text { coupling efficiency }\end{array}$ & $\begin{array}{l}\text { The fiber end on the ferrule is not } \\
\text { well-polished }\end{array}$ & Polish the fiber again \\
\hline 39 & Low coupling efficiency & $\begin{array}{l}\text { The three screws on the adapter are not } \\
\text { well-adjusted }\end{array}$ & $\begin{array}{l}\text { Adjust the screws more carefully. } \\
\text { Can start with a larger core, bigger NA fiber, } \\
\text { then switch to the thinner fiber }\end{array}$ \\
\hline 44 & No modulation found & $\begin{array}{l}\text { Low expression efficacy; miss injected } \\
\text { location; low light delivery efficacy; bad } \\
\text { electrode }\end{array}$ & $\begin{array}{l}\text { Test the expression in a rat; record from the } \\
\text { correct position; check the output power; } \\
\text { check the impedance of the electrode }\end{array}$ \\
\hline
\end{tabular}

expression in the primate brain after all the experiments have been completed.

In two cases, we performed histological studies of the opsin expression in nonhuman primates. The animals were perfused with $2 \%$ paraformaldehyde and their brains were extracted and sent to NeuroScience Associates (Knoxville, Tennessee) for histological slicing and anti-YFP immunostaining. In both monkeys, the expression was effective within a cylindrical cortical volume of $\sim 2 \mathrm{~mm}$ in diameter. Example of histological sections from a rat and a monkey are shown in Fig. 6.

\section{Discussion}

\subsection{Protocol Improvement}

Here, we have described our procedures in setting up basic optogenetic experiments in nonhuman primates, which have proven to be highly reliable and replicable. The improvement of these procedures mainly includes two key steps: the "in-chair" injection and the use of a tapered, thinner fiber.

The "in-chair" injection highly improves the injection efficacy and flexibility. The basic requirement assumes that the monkey has been trained to sit in the chair with its head in restraint for a few hours and has a chamber ready for recording. Then, injection can be performed in a way similar to regular recording. On the other hand, given that the process could last for several hours with the potential to cause discomfort and result in agitation, minor movements of the injection needle were unavoidable even if we tried to secure the chair in a rigid way. Although we found this did not affect the efficacy of the injection, precautions similar to those used when conducting electrophysiological recordings should be taken to prevent the monkey from moving. The variety of chairs and restraints utilized by different labs must be considered when using our protocol, but we have found the following elements to be essential. When mounting the stereotaxic rail on the chair, be aware of the distance between the rail and the monkey head. The positioning of the rail should allow the injection needle to access the target area. Theoretically, the rail does not need to be mounted on the chair. Any rigid frame near the monkey head could serve as a base. When a chamber is not available, or chronic implantation (i.e., array) is needed, the injection system can be used in the OR setting without any modification except discarding the rail on the chair and using a standard stereotaxic apparatus. Compared to the "in-chair" injection, such injection in the OR with monkey sedated would avoid any possible issues associated with movement.

As an acute readout device, the size of the optrode should be as small as possible for repeated penetrations into the brain. The optrode we described here was made from the smallest size (to the best of our knowledge) of commercially available optic fiber and electrode, rendering a diameter decrease to around $200 \mu \mathrm{m}$. In addition, we pull the fiber to taper the tip rather than leaving it blunt, which we believe makes it less likely to cause cortical 
(a)

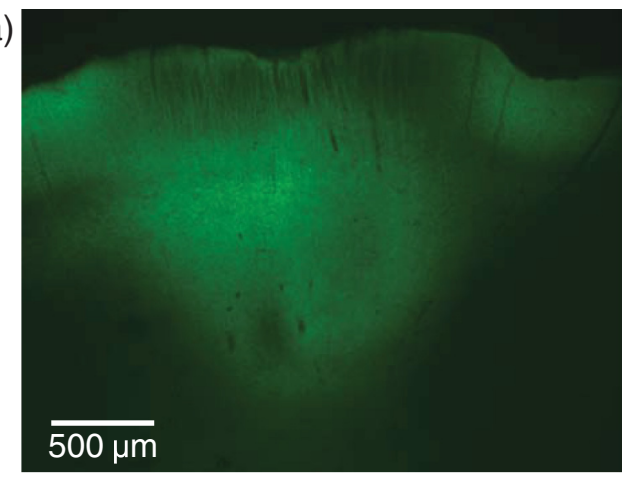

(b)

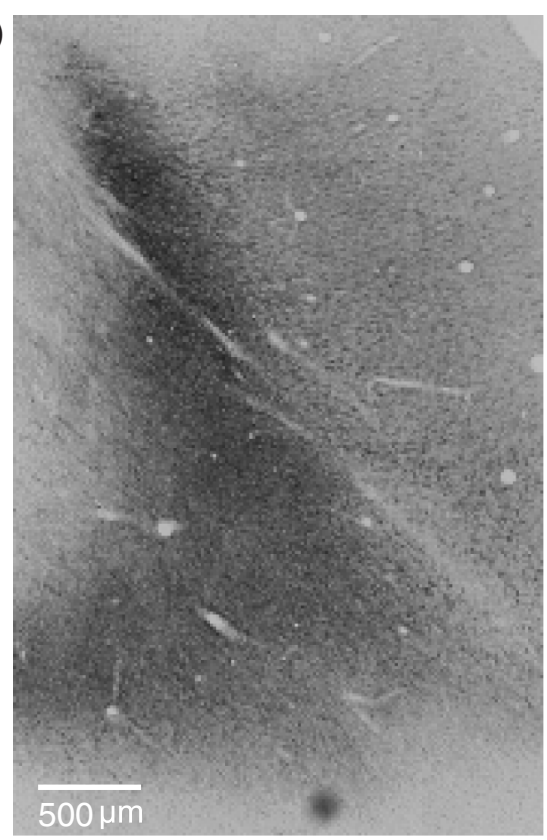

Fig. 6 Histology. (a) A histological section from a rat showing the extent of opsin expression. The green colors are the fluorescence due to YFP expression. (b) The same virus was successfully expressed also in a primate where the dark colors indicate the level of anti-YFP labeling due YFP expression.

damage. Even so, such two-tip devices are not optimal in terms of reducing tissue damage. A device that can deliver light and record a neural signal at the same time but share a single tip is therefore more ideal. Thus, the coaxial optrode ${ }^{21}$ could be a better choice.

Alternatively, chronically implanting an optic fiber with a readout device could be another direction to consider, since the extent of tissue damage will be minimized with a chronic optrode. We note that an example of a chronic device, in which a tapered fiber is integrated with a Utah array, has been successfully applied to rodents and nonhuman primates.$^{25}$ Such an optoarray has advantages over a single optrode in that it allows simultaneous recording in larger spatial scale, which will be particularly useful in studies of spatiotemporal activity features of neural circuitry with single-site stimulation and setting up certain computational models. Ideally, an opto-electrode array with spatiotemporally controlled multisite light delivery capabilities would be ideal to explore the relationship between neural activity and behavior.

\subsection{Experimental Consideration}

To advance beyond the interest in primate optogenetics as merely a novel technique, one critical question is how much it can affect an animal's behavior. These behavioral results are important for establishing that optogenetics might prove useful for designing clinical treatments. To address this, several recent studies have applied optogenetic stimulation in the nonhuman primate while measuring behavioral effects caused by stimulating certain areas. ${ }^{6-10,12}$ Under the general framework of "writing in" signals to program behavior, so far the majority of observed effects have been on oculomotor movements. Saccades could be evoked by optogenetically stimulating V1, and possibly in FEF, ${ }^{9}$ but not in LIP. ${ }^{10}$ Optogenetic inactivation of superior colliculus also caused deficits in the metrics of saccadic eye movements. ${ }^{8}$ However, other groups were unable to induce body movements (such as hand reaching) by stimulating cortical motor and premotor areas. ${ }^{5}$ We found that optogenetic stimulation in a higher cortical area like LIP did not affect simple visually guided saccades; however, when the animal was involved in a complex discrimination task that may rely on attention or salience detection, optogenetic stimulation was as effective as electrical microstimulation in biasing the animal's choice. ${ }^{10}$ Another study in $\mathrm{FEF}^{6}$ also found that optogenetic stimulation affected saccade latency in a complex visual-guided saccade task. In addition, recently it has been demonstrated that optogenetic stimulation of the somatosensory cortex can be reliably detected in a sensory detection task. ${ }^{12}$ What we learn from these findings is that optogenetic stimulation may be more effective if such "write in" information can be combined with intrinsic signals and serve as a modulator to affect network activity. However, the stimulation itself-at least at a single site—is usually not powerful enough to serve as a signal source to execute an action, especially in higher cortical areas. As an alternative hypothesis, any simple behavior requires the integrated cooperation of several regions, and that simply changing a tiny part of it will not result in a direct behavioral change. Further study will be needed to decipher the brain's programming strategy to render reprogramming possible.

\subsection{Summary}

The protocol described here aids in helping people with either limited resources or experience in optogenetics to set up their own optogenetic experiments in the nonhuman primate. The basic goal is to successfully transduce regions in the primate brain and readout the optically induced neural modulation. Beyond this, researchers should set more specific goals in applying optogenetics and design more specific experiments to incorporate this technique. 


\section{Acknowledgments}

We thank John Ghenne for his expert assistance with animal care and training, the Deisseroth laboratory for graciously providing the viral constructs used in the experiments, and the Shenoy laboratory, especially Dan O'Shea and Werapong Goo, for graciously sharing their experiences. We also thank Jing Wang for her help in developing the protocol. This work was supported by DARPA REPAIR (N66001-10-C-2010).

\section{References}

1. A. M. Aravanis et al., "An optical neural interface: in vivo control of rodent motor cortex with integrated fiberoptic and optogenetic technology," J. Neural Eng. 4, S143-S156 (2007).

2. A. R. Adamantidis et al., "Neural substrates of awakening probed with optogenetic control of hypocretin neurons," Nature 450, 420-424 (2007).

3. M. E. Carter et al., "Tuning arousal with optogenetic modulation of locus coeruleus neurons," Nat. Neuroscience 13, 1526-1533 (2010).

4. X. Han et al., "Millisecond-timescale optical control of neural dynamics in the nonhuman primate brain," Neuron 62, 191-198 (2009).

5. I. Diester et al., "An optogenetic toolbox designed for primates," Nat. Neuroscience 14, 387-397 (2011).

6. A. Gerits et al., "Optogenetically induced behavioral and functional network changes in primates," Curr. Biol. 22, 1722-1726 (2012).

7. M. Jazayeri, Z. Lindbloom-Brown, and G. D. Horwitz, "Saccadic eye movements evoked by optogenetic activation of primate V1," Nat. Neuroscience 15, 1368-1370 (2012).

8. J. Cavanaugh et al., "Optogenetic inactivation modifies monkey visuomotor behavior," Neuron 76, 901-907 (2012)

9. S. Ohayon et al., "Saccade modulation by optical and electrical stimulation in the macaque frontal eye field," J. Neurosci. 33, 16684-16697 (2013).

10. J. Dai, D. I. Brooks, and D. L. Sheinberg, "Optogenetic and electrical microstimulation systematically bias visuospatial choice in primates," Curr. Biol. 24, 63-69 (2014).

11. X. Han et al., "A high-light sensitivity optical neural silencer: development and application to optogenetic control of non-human primate cortex," Front Syst. Neurosci. 5, 18 (2011).

12. T. May et al., "Detection of optogenetic stimulation in somatosensory cortex by non-human primates-towards artificial tactile sensation," PLoS One 9, e114529 (2014).

13. A. Galvan et al., "In vivo optogenetic control of striatal and thalamic neurons in non-human primates," PLoS One 7, e50808 (2012).

14. http://www.med.unc.edu/genetherapy/vectorcore.

15. http://www.med.upenn.edu/gtp/vectorcore.

16. O. Yizhar et al., "Optogenetics in neural systems," Neuron 71, 9-34 (2011).

17. L. Fenno, O. Yizhar, and K. Deisseroth, "The development and application of optogenetics," Аппи. Rev. Neurosci. 34, 389-412 (2011).

18. J. Mattis et al., "Principles for applying optogenetic tools derived from direct comparative analysis of microbial opsins," Nat. Methods $\mathbf{9}$, 159-172 (2011).

19. O. Yizhar et al., "Neocortical excitation/inhibition balance in information processing and social dysfunction," Nature 477, 171-178 (2011).

20. K. M. Tye and K. Deisseroth, "Optogenetic investigation of neural circuits underlying brain disease in animal models," Nat. Rev. Neurosci. 13, 251-266 (2012)

21. I. Ozden et al., "A coaxial optrode as multifunction write-read probe for optogenetic studies in non-human primates," J. Neurosci. Methods 219, 142-154 (2013).

22. X. Han, "Optogenetics in the nonhuman primate," Prog. Brain Res. 196, 215-233 (2012).
23. F. Wu et al., "An implantable neural probe with monolithically integrated dielectric waveguide and recording electrodes for optogenetics applications," J. Neural Eng. 10, 056012 (2013).

24. T. I. Kim et al., "Injectable, cellular-scale optoelectronics with applications for wireless optogenetics," Science 340, 211-216 (2013).

25. J. Wang et al., "Approaches to optical neuromodulation from rodents to non-human primates by integrated optoelectronic devices," in Proc. 2011 Int. Conf. on IEEE Engineering Medicine and Biology Society, pp. 7525-7528, (2011).

Ji Dai obtained his PhD degree from Institute of Biophysics, Chinese Academy of Sciences, and then worked as a postdoctoral fellow in the Laboratory of David Sheinberg at Brown University. He is now an associate investigator at the Institute of Psychology, Chinese Academy of Sciences.

Ilker Ozden obtained his $\mathrm{PhD}$ degree in physics from Brown University. He worked as a postdoctoral fellow in the laboratory of Samuel S.-H. Wang at Princeton University. He is now an assistant professor of engineering (research) at Brown University.

Daniel I. Brooks is a postdoctoral fellow and lecturer at Tufts University, where he studies comparative cognition and avian vision. He previously worked as a postdoctoral fellow with David Sheinberg at Brown University.

Fabien Wagner obtained his BS degree in mathematics/physics/ engineering from the Ecole Polytechnique in France (2009) and his $\mathrm{PhD}$ degree in neuroscience/neuroengineering from Brown University in the United States (2014). He is now a postdoctoral research scientist at the Ecole Polytechnique Fédérale de Lausanne in Switzerland.

Travis May is a PhD candidate in the Center for Biomedical Engineering at Brown University working in the neuroengineering laboratory of Dr. Arto Nurmikko.

Naubahar S. Agha is a PhD student at the Center for Biomedical Engineering at Brown University.

Benjamin Brush is an MD candidate at Harvard Medical School. He previously worked as a research assistant under Arto Nurmikko at Brown University.

David Borton received his BS degree in biomedical engineering from Washington University in St. Louis in 2006. He then joined Brown University, where he worked towards his PhD degree developing implantable, wireless, and broadband neural interfaces for both basic neuroscience and clinical applications, and received his $\mathrm{PhD}$ degree in 2012. He then continued his neuroengineering research at the Swiss National Institute of Technology as a postdoctoral fellow. He returned to Brown as an assistant professor in 2014.

Arto V. Nurmikko is a L. Herbert Ballou University professor of engineering and physics at Brown. He received his degrees from the University of California, Berkeley, with postdoctoral stays at MIT and Hebrew University. He is a fellow of the American Physical Society, fellow of the Institute of Electrical and Electronics Engineers, fellow of the Optical Society of America, and a member of the American Academy of Arts and Sciences.

David L. Sheinberg is a professor of Neuroscience at Brown University. He received his BA degree from Yale and his $\mathrm{PhD}$ degree from Brown. Following postdoctoral fellowships at Baylor and the Max Planck Institute, he returned to Brown University as a faculty member in the Department of Neuroscience in 2000. He is the director of the Neuroscience Graduate Program. 